\title{
Análisis del patrón de consumo de cannabis en estudiantes universitarios
}

\author{
Sílvia Font-Mayolas*; María Eugenia Gras** ; Montserrat Planes*** \\ Instituto de Investigación sobre Calidad de Vida. Universidad de Girona. \\ * Profesora Lectora del Área de Personalidad, Evaluación y Tratamiento Psicológico de la Universidad de Girona \\ * Profesora Titular del Área de Metodología de las Ciencias del Comportamiento de la Universidad de Girona. \\ ** Profesora Titular del Área de Personalidad, Evaluación y Tratamiento Psicológico de la Universidad de Girona.
}

\section{RESUMEN}

Se analiza el patrón de consumo de cannabis en una muestra de 580 jóvenes universitarios (88.4\% mujeres, edad media 21.4 años). Si bien se observa que proporcionalmente más hombres que mujeres consumen habitualmente cannabis, la magnitud de consumo de los que se reconocen usuarios es similar en ambos sexos. Se detecta que la dependencia percibida al cannabis se incrementa en función de la magnitud de consumo. Existe una relación positiva entre consumo de tabaco y de cannabis: el $90 \%$ de los jóvenes que no ha probado nunca el tabaco tampoco ha probado nunca el cannabis, mientras que menos del $15 \%$ de los fumadores habituales de tabaco se hallan en esa situación. Estos resultados sugieren la necesidad de abordajes conjuntos para la prevención del consumo de drogas, principalmente en lo que se refiere al tabaco y el cannabis.

Palabras clave: consumo de cannabis, dependencia percibida, consumo de tabaco, estudiantes universitarios.

\section{ABSTRACT}

The patterns of cannabis use amongst a sample of 580 university students (88.4\% female, mean age 21.4 years old) were analysed. Proportionally more males than females normally consume cannabis, although there was no difference in the amount of consumption by gender. Perceived dependence on cannabis increased with the amount of consumption. There was also a positive relationship between tobacco and cannabis use, such that $90 \%$ of students who had never tried tobacco, had also never tried cannabis. Conversely, less than $15 \%$ of tobacco smokers have never tried cannabis. These findings suggest the need for combined actions to prevent drug consumption, particularly with regards to tobacco and cannabis

Key words: cannabis consumption, perceived dependence, tobacco consumption, university students.

\section{INTRODUCCIÓN}

$\mathbf{L}$ os derivados de Cannabis sativa o cannabinoides, también conocidos como hachís y marihuana, son el grupo de drogas ilegales más consumidas en la población general española. Desde 1995 hasta 2001 se ha doblado el porcentaje de españoles entre 15 y 64 años que informan haber consumido cannabinoides en los últimos 30 días (3.1\% a 6.5\% respectivamente) (Ministerio del Interior, 2003).

La edad media de inicio del consumo de cannabis en población general española en el año 2001 se situó en los 18.4 años siendo más precoces los hombres (18.2 años) que las mujeres (18.7 años) (Ministerio del Interior, 2003). En estudiantes españoles de 14-18 años en el año 2000 esa edad media fue de 14.8 años
(14.7 años en los chicos y 14.9 años en las chicas) (Ministerio del Interior, 2002).

En cuanto al consumo de cannabis en población universitaria, durante el curso académico 1996-97 se realizó un estudio con una muestra de 410 alumnos de Ciencias de la Salud de la Universidad de Oviedo (edad media $=21.5$ años). Se detectó una prevalencia de consumo de cannabis en los últimos 12 meses del $19 \%$ y en el último mes del $8.5 \%$. Únicamente se encontraron diferencias en función del sexo en el consumo en el último mes, presentando las mujeres una tasa significativamente inferior a la de los hombres. La edad media de inicio para el conjunto de los estudiantes fue de 17.5 años, sin diferencias en función del sexo (Bobes, Bascarán, González y Sáiz, 2000). 
Las principales motivaciones que conducen a probar el cannabis registradas en jóvenes españoles de 14-18 años son: la curiosidad (89.9\%), la necesidad de sentir nuevas sensaciones (35.5\%) y la diversión (30.8\%) (Ministerio del Interior, 2002). Amigó y Seshadri (1999) realizaron un estudio con una muestra de 156 jóvenes valencianos consumidores frecuentes de cannabis que incluía a 58 alumnos de bachillerato y COU y a 90 estudiantes de primero de carrera de Psicología y Magisterio (media de edad de la muestra total $=18.32$ años y DT $=3.48$ ). Las principales motivaciones informadas para el consumo de cannabis fueron: experimentar sensaciones nuevas y diferentes (54\%) o placer (41\%), "desconectar" de los problemas emocionales (47\%), sentirse "colocado" (46\%) y disfrutar más de la diversión (40\%). Chabrol, Ducongé, Casas, Roura y Carey (2005) estudiaron los motivos del consumo de cannabis en una muestra formada por 212 estudiantes franceses de 15-22 años de edad mediante la escala Marihuana Motives Measure de Simons et al. (1998; cf. Chabrol et al., 2005). Se observó que la subescala de Intensificación ("enhancement"), que incluye ítems del tipo "Yo consumo marihuana porque es excitante" ("I use marijuana because it's exciting"), resultaba un predictor del consumo de cannabis en los chicos. Asimismo se detectó que la subescala de Expansion ("expansion"), que incorpora ítems del tipo "Yo consumo marihuana para expandir mis horizontes" ("I use marijuana to expand my awareness"), era predictora del consumo de cannabis en chicas.

En la actualidad, el cannabis se ha convertido en un controvertido tema de debate debido a que se reciben mensajes antagónicos. Por una parte se informa de las posibles beneficiosas aplicaciones clínicas del cannabis y por otra parte se hace referencia a que no es una sustancia inocua (Fuentes, Ruiz-Gayo, Manzanares, Vela, Reche y Corchero, 1998).

La percepción de riesgo (la proporción de estudiantes españoles de 14-18 años que considera que el consumo de cannabis puede suponer bastantes o muchos problemas para la salud o de otro tipo) se sitúa en un $38.8 \%$ en caso de consumo esporádico (una vez al mes o menos frecuentemente) y en un $77.1 \%$ en caso de consumo habitual (una vez por semana o más frecuentemente). El riesgo asociado al consumo habitual de cannabis es algo superior al atribuido al consumo diario de tabaco $(73.3 \%)$ e inferior al atribuido al consumo habitual de otras drogas ilegales: éxtasis (85.2\%), cocaína (88.3\%) y heroína (88.9\%) (Ministerio del Interior, 2002). En un trabajo de Calafat, Fernández, Becoña, Gil, Juan y Torres (2000) sobre consumo de cannabis mediante una muestra de 1341 jóvenes (media de edad = 22.6 años, DT $=5$ ) se observó que a mayor frecuencia de uso corresponde una menor percepción del riesgo asociado al consumo de sustancias. En un estudio de Cunill, Planes y Gras (1998) se evaluaron las creencias de los estudiantes univer- sitarios respecto a la dureza de las drogas mediante autoinformes con una muestra de 155 participantes (media de edad $=21.48$; DT $=3.35$ ). Se detectaron notables diferencias de creencias en la dureza respecto a la marihuana en función del sexo de manera que los hombres mayoritariamente consideran que la marihuana no es una droga dura $(72.7 \%)$ en comparación a las mujeres (53.4\%). En una investigación de Megías (2004) sobre la percepción social de los problemas de las drogas en población española a través de entrevistas con una muestra de 1700 participantes de 15 a 65 años, se observó que el cannabis es la droga que se percibe como menos peligrosa (3.69 en una escala de peligro creciente de 1 a 5) por debajo tanto de las drogas legales (tabaco $=3.71 \mathrm{y}$ alcohol $=3.76$ ) como de las ilegales (hipno-sedantes $=4.28$ anfetaminasalucinógenos-éxtasis $=4.53$ cocaína $=4.74$ y heroína = 4.86).

Por otra parte, los consumos de alcohol, tabaco y cannabis suelen presentar una asociación muy estrecha de manera que el consumo de una de estas sustancias se asocia con el consumo de las demás. Es decir, la proporción de estudiantes españoles de 1418 años que consumieron cannabis en los últimos 12 meses aparejado al consumo de tabaco fue del $73.7 \%$ y al alcohol del 97.8\% (Ministerio del Interior, 2002). En la misma línea, en un estudio sobre consumo de drogas de síntesis en una muestra de 1597 estudiantes de secundaria asturianos (media de edad = 16 años, DT = 1.29) se detectó que el $60.5 \%$ de los consumidores de pastillas las acompañaban de cannabis (Fernández et al., 2003). De hecho, el consumo de drogas legales podría facilitar el posterior consumo de marihuana que a su vez abriría la puerta al consumo de otras drogas ilegales según el modelo de la escalada (Kandel y Yamaguchi, 1985).

Siguiendo las propuestas de investigación científica del Grupo de Estudios sobre el Cannabis (Becoña et al., 2004), los principales objetivos de esta investigación son:

1. Describir el patrón de consumo de cannabis en una muestra de estudiantes universitarios de Girona en función del sexo.

2. Conocer el consumo diferencial de cannabis según la variable sexo.

3. Evaluar la relación entre la edad inicial de experimentación con esta sustancia y el estatus actual de consumo.

4. Estudiar hasta qué punto están extendidas ciertas creencias sobre los beneficios del consumo de cannabis.

5. Evaluar la dependencia percibida al cannabis y su relación con la magnitud de consumo. 
6. Analizar la relación entre el consumo de cannabis y el consumo de tabaco.

\section{MÉTODO}

\section{Participantes}

Participan en el estudio los estudiantes matriculados en la Facultad de Educación y Psicología (FEP) de la Universidad de Girona durante el curso académico 2004-05 que estaban presentes el día de la recogida de datos. Inicialmente se recogió información de 607 estudiantes, de los cuales 27 fueron eliminados por no rellenar adecuadamente el cuestionario. El tipo de estudios que se imparten desde la Facultad (Magisterio, Psicología, etc.) suelen ser cursados mayoritariamente por mujeres; de ahí que de los 580 estudiantes de la muestra final, el $88.3 \%$ sean mujeres y el $11.2 \%$ hombres. Tres participantes no informan sobre este dato. La edad media de los participantes es de 21.4 años (DT $=4.4)$. El porcentaje de participación es del $95.5 \%$.

\section{Diseño e Instrumento}

Se realizó un estudio transversal de encuesta. La información se recogió a partir de un cuestionario de elaboración propia mediante el cual se evaluaron las variables que se enumeran a continuación:

- Edad.

- Sexo.

- Patrón de consumo de cannabis. Se adapta la pregunta creada por Becoña, Palomares y García (1994) substituyendo la palabra "cigarrillos" por "porros". Se solicita indicar la frase que más se ajuste al caso entre las siguientes opciones: "no he fumado nunca un porro entero", "he fumado un porro o más sólo para probar", "antes fumaba porros, pero ya no fumo", "fumo, pero sólo un porro al mes", "fumo, pero sólo un porro a la semana" y "fumo más de un porro a la semana".

- Magnitud de consumo de cannabis. Al igual que en el trabajo de Pinazo, Pons y Carreras (2002) se pregunta por el número de porros consumidos en el último mes. Las alternativas de respuesta son: "1-5", "6-9" y "10 o más".

- Edad de prueba del primer porro. Se formula la pregunta "¿a qué edad probaste por primera vez el porro?".

- Creencias sobre los beneficios del consumo de cannabis. Se solicita a los participantes que indiquen si son verdaderas o falsas las siguientes fra- ses: "creo que el porro ayuda a relajarse" y "creo que el porro ayuda a desinhibirse"

- Dependencia percibida al consumo de cannabis. Se solicita indicar cuál de las siguientes situaciones se corresponde al caso del participante: "estoy muy enganchado al porro. Me costaría mucho dejarlo", "estoy enganchado al porro pero lo puedo dejar cuando quiera" y "no estoy enganchado al porro".

- Patrón de consumo de tabaco. Se utiliza la pregunta elaborada por Becoña et al. (1994) según la cual se propone escoger la frase que más se ajuste al caso entre las siguientes alternativas: "no he fumado nunca un cigarrillo entero," "he fumado un cigarrillo o más sólo para probar", "antes fumaba pero ya no fumo," "fumo, pero sólo un cigarrillo al mes", "fumo, pero sólo un cigarrillo a la semana", "fumo más de un cigarrillo a la semana" y "fumo diariamente".

\section{Procedimiento}

La recogida de datos se realizó en cada uno de los cursos de los estudios que se imparten desde la FEP (Psicología, Psicopedagogía, Educación Social, Pedagogía y Magisterio).

Las responsables de la investigación contactaron con uno de los profesores correspondientes para concretar una fecha de administración del cuestionario. Se solicitó la colaboración voluntaria y anónima de los estudiantes presentes.

\section{Análisis de datos}

Los datos se analizaron mediante el paquete estadístico SPSS versión 13.0. Para el análisis comparativo entre hombres y mujeres o entre consumidores según su estatus, se utilizó la prueba chi-cuadrado cuando la variable era categórica y la prueba " $\mathrm{t}$ " de comparación de medias en el caso de variables numéricas. Para comparar la edad de primera experiencia con el cannabis, según el sexo y el estatus de consumidor, se hizo uso del análisis de la variancia. En todos los casos el nivel de significación fue de 0,05.

\section{RESULTADOS}

\section{Descripción del patrón de consumo de cannabis}

En la Tabla 1 se presenta la distribución de los participantes en función del patrón de consumo de cannabis y el sexo. Tres de cada diez hombres y dos de cada diez mujeres son en la actualidad consumidores de esta sustancia. El consumo experimental también es superior entre los hombres. Casi la mitad de las 
participantes no ha probado nunca el cannabis, mientras que menos de la cuarta parte de los hombres se hallan en esa situación. Las diferencias observadas son estadísticamente significativas $\left(\chi^{2}(3)=15.15\right.$; $\mathrm{p}=0.002)$; para el cálculo de este índice se han agrupado todos los participantes que informan consumir cannabis en la actualidad, con independencia de la frecuencia de consumo.

Tabla 1. Distribución de los participantes según el patrón de consumo de cannabis y el sexo

\begin{tabular}{|l|c|c|}
\hline $\begin{array}{l}\text { Consumo de } \\
\text { cannabis }\end{array}$ & Hombres \% (n) & Mujeres \% (n) \\
\hline $\begin{array}{l}\text { No he fumado } \\
\text { nunca un porro } \\
\text { entero. }\end{array}$ & $23.1(15)$ & $48.2(247)$ \\
\hline $\begin{array}{l}\text { He fumado un } \\
\text { porro o más sólo } \\
\text { por probar. }\end{array}$ & $30.8(20)$ & $22,3(114)$ \\
\hline $\begin{array}{l}\text { Antes fumaba } \\
\text { porros pero ya } \\
\text { no fumo. }\end{array}$ & $15.4(10)$ & $8.8(45)$ \\
\hline $\begin{array}{l}\text { Fumo sólo un } \\
\text { porro al mes. }\end{array}$ & $4.6(3)$ & $5.1(26)$ \\
\hline $\begin{array}{l}\text { Fumo sólo } \\
\text { un porro a la } \\
\text { semana. }\end{array}$ & $9.2(6)$ & $4.3(22)$ \\
\hline $\begin{array}{l}\text { Fumo más de } \\
\text { un porro a la } \\
\text { semana. }\end{array}$ & $16.9(11)$ & $11.4(58)$ \\
\hline
\end{tabular}

\section{Consumo de cannabis en función de sexo}

En la Tabla 2 se puede hallar la distribución de los consumidores de cannabis según la magnitud de consumo en el último mes y el sexo. A pesar de que los hombres informan haber fumado más porros en el último mes que las mujeres, las diferencias observadas no son estadísticamente significativas $\left(\chi^{2}(2)=1\right.$; $\mathrm{p}=0.61)$.

Tabla 2. Distribución de los hombres y mujeres según la magnitud de consumo de cannabis en el último mes.

\begin{tabular}{|l|c|c|}
\hline $\begin{array}{l}\text { Consumo } \\
\text { de cannabis } \\
\text { durante } \\
\text { el último mes }\end{array}$ & Hombres \%(n) & Mujeres \% (n) \\
\hline $\begin{array}{l}\text { Entre 1 y } 5 \\
\text { porros }\end{array}$ & $27.3(3)$ & $43.3(26)$ \\
\hline $\begin{array}{l}\text { Entre 6 y } 9 \\
\text { porros }\end{array}$ & $18.2(2)$ & $15(9)$ \\
\hline Más de 9 porros & $54.5(6)$ & $41.7(25)$ \\
\hline
\end{tabular}

Edad inicial de experimentación con el cannabis y consumo actual

La edad media de la primera experiencia con el cannabis es de 16.1 años (DT = 1.8). Por sexos, las mujeres se iniciaron antes (Media = 16 años; D.T = 1.8) que los hombres (Media $=16.5$ años; DT $=2$ ). Se analizó esta variable en función del estatus de consumo de cannabis. Para ello se clasificó a los participantes en tres grupos: aquellos que fumaron un porro o más pero sólo por probar (no fumadores), los que a pesar de haber fumado habitualmente durante algún tiempo, en la actualidad no fuman (exfumadores), y los que son consumidores ocasionales o habituales de esta sustancia (fumadores). Las medias y desviaciones típicas de la edad en que probaron por primera vez el cannabis en función del estatus actual de consumidor y el sexo se muestran en la Tabla 3. Se aprecia que los que sólo probaron esta sustancia (no fumadores) lo hicieron a una edad superior que aquellos que en algún momento la consumieron con regularidad (exfumadores y fumadores). El resultado del análisis de la varianza del diseño de dos factores (sexo/estatus de consumidor) indica que el efecto de interacción entre el sexo y el estatus de consumidor no es estadísticamente significativo $(F(2,307)=1.02 ; p=0.36)$, pero sí el efecto principal tanto del sexo $(F(1.307)=3.95 ; p$ $=0.05)$ como del estatus de consumidor $(F(2,307)=$ 9.86; $p<0.0005)$. En este sentido, las mujeres son significativamente más precoces que los hombres. En cuanto al estatus de consumo, los resultados de los contrastes de Scheffé indican que los exfumadores y fumadores actuales de cannabis son más precoces que los que sólo lo han probado ( $<<0.0005$ y $p=0.001)$, pero no existen diferencias significativas entre exfumadores y fumadores de esta sustancia respecto a la edad de inicio de consumo $(p=0.54)$.

Tabla 3. Edad media de la primera experiencia con el cannabis según el estatus de consumo actual y el sexo.

\begin{tabular}{|l|c|c|}
\hline $\begin{array}{l}\text { Estatus actual } \\
\text { de consumidor }\end{array}$ & $\begin{array}{c}\text { Hombres } \\
\text { Media (DT) }\end{array}$ & $\begin{array}{c}\text { Mujeres } \\
\text { Media (DT) }\end{array}$ \\
\hline $\begin{array}{l}\text { No fumador de } \\
\text { cannabis }\end{array}$ & $\begin{array}{c}17.5(2,2) \\
\mathrm{n}=20\end{array}$ & $\begin{array}{c}16.5(1.9) \\
\mathrm{n}=112\end{array}$ \\
\hline $\begin{array}{l}\text { Exfumador de } \\
\text { cannabis. }\end{array}$ & $\begin{array}{c}15.9(1.8) \\
\mathrm{n}=10\end{array}$ & $\begin{array}{c}15.4(1.3) \\
\mathrm{n}=45\end{array}$ \\
\hline $\begin{array}{l}\text { Fumador habitual } \\
\text { u ocasional de } \\
\text { cannabis. }\end{array}$ & $\begin{array}{c}15.9(1.5) \\
\mathrm{n}=20\end{array}$ & $\begin{array}{c}15.8(1.8) \\
\mathrm{n}=106\end{array}$ \\
\hline
\end{tabular}

\section{Creencias sobre los beneficios del consumo de cannabis}

En la Figura 1 se muestran los porcentajes de participantes que consideran verdaderas dos afirmaciones 
sobre el consumo de cannabis: "que ayuda a relajarse" y "que ayuda a desinhibirse". Nueve de cada diez fumadores de cannabis, y algo más de siete de cada diez exfumadores creen que el consumo de esta sus- tancia ayuda a relajarse. Menos de la mitad de los no fumadores también lo creen. Las diferencias observadas son estadísticamente significativas $\left(\chi^{2}(2)=77.4\right.$; $\mathrm{p}<0.0005)$.

Figura 1.

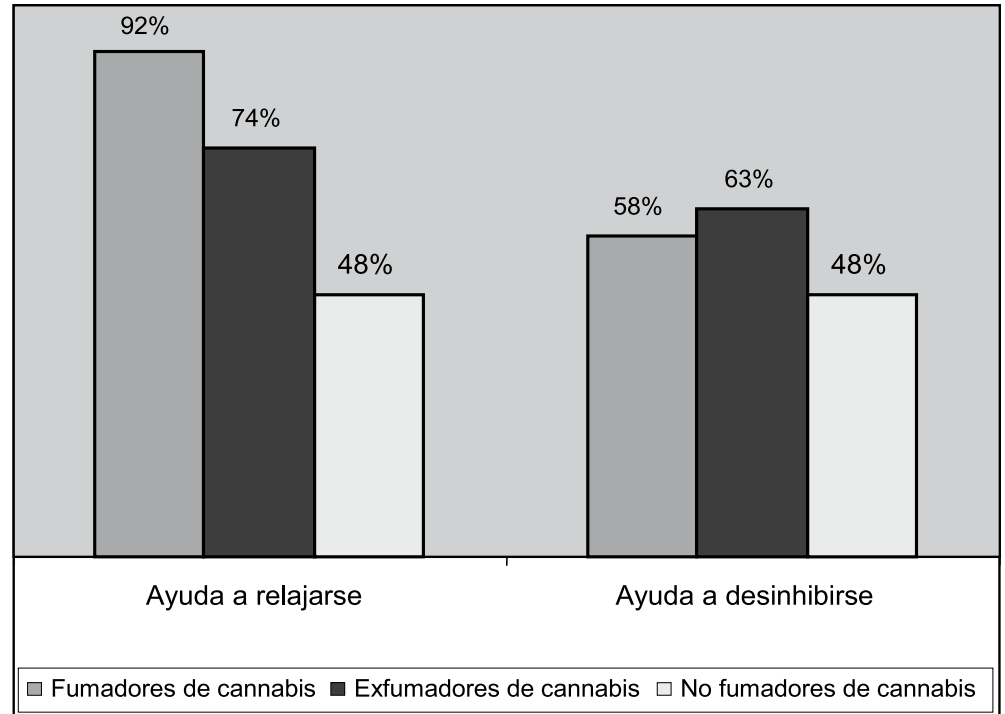

Porcentaje de participantes que consideran verdaderas cada una de las afirmaciones sobre las consecuencias del cannabis, según el estatus de consumo actual.

El mayor porcentaje de participantes que cree que fumar ayuda a desinhibirse se halla entre los exfumadores. De nuevo son los no fumadores el grupo donde con menor frecuencia se tiene esta creencia $\left(\mathrm{X}^{2}(2)=\right.$ 7; $p=0.03$ ).

\section{Dependencia percibida al cannabis y magnitud de consumo}

La distribución de los consumidores habituales de cannabis según la dependencia percibida a esta sustancia y la magnitud de consumo en el último mes aparece en la Tabla 4. Se aprecia que la dependencia percibida al cannabis se incrementa en función de la magnitud del consumo. Ninguno de los participantes de bajo consumo (entre 1 y 5 porros al mes) cree estar "muy enganchado" y menos de un 11\% cree estar "enganchado". Estos porcentajes van en aumento a medida que se incrementa la magnitud del consumo. Las diferencias observadas son estadísticamente significativas $\left(X^{2}(2)=17.18 ; P<0.0005\right)$. Para el cálculo del índice chi-cuadrado se agruparon las categorías "muy enganchado" y "enganchado" para evitar el incumplimiento de las condiciones de aplicación de la prueba estadística.

Tabla 4. Dependencia percibida al cannabis y magnitud de consumo entre los consumidores habituales de esta sustancia.

\begin{tabular}{|c|c|c|c|c|}
\hline $\begin{array}{l}\text { Consumo de cannabis en el } \\
\text { último mes }\end{array}$ & $\begin{array}{l}\text { Estoy muy "enganchado" al } \\
\text { porro. Me costaría mucho } \\
\text { dejarlo. }\end{array}$ & $\begin{array}{l}\text { Estoy "enganchado" al } \\
\text { porro pero lo puedo dejar } \\
\text { cuando quiera. }\end{array}$ & $\begin{array}{l}\text { No estoy "enganchado" } \\
\text { al porro. }\end{array}$ & Total \\
\hline Entre 1 y 5 porros & $\begin{array}{c}0 \% \\
{[0 \%]} \\
(0)\end{array}$ & $\begin{array}{c}10.7 \% \\
{[16.7 \%]} \\
\text { (3) }\end{array}$ & $\begin{array}{c}89.3 \% \\
{[59.5 \%]} \\
(25)\end{array}$ & $\begin{array}{c}100 \% \\
{[41.8 \%]} \\
(28)\end{array}$ \\
\hline Entre 6 y 9 porros & $\begin{array}{c}9.1 \% \\
{[14.3 \%]} \\
(1)\end{array}$ & $\begin{array}{c}27.3 \% \\
{[16.7 \%]} \\
\text { (3) }\end{array}$ & $\begin{array}{c}63.6 \% \\
{[16.7 \%]} \\
(7)\end{array}$ & $\begin{array}{c}100 \% \\
{[16.4 \%]} \\
(11)\end{array}$ \\
\hline Más de 9 porros. & $\begin{array}{c}21.4 \% \\
{[85.7 \%]} \\
(6) \\
\end{array}$ & $\begin{array}{c}42,9 \% \\
{[66.7 \%]} \\
(12) \\
\end{array}$ & $\begin{array}{c}35.7 \% \\
{[23.8 \%]} \\
(10) \\
\end{array}$ & $\begin{array}{c}100 \% \\
{[41.8 \%]} \\
(28)\end{array}$ \\
\hline Total & $\begin{array}{c}10.4 \% \\
{[100 \%]} \\
(7)\end{array}$ & $\begin{array}{c}26.9 \% \\
{[100 \%]} \\
(18)\end{array}$ & $\begin{array}{c}62,7 \% \\
{[100 \%]} \\
(42)\end{array}$ & $\begin{array}{c}100 \% \\
{[100 \%]} \\
(67)\end{array}$ \\
\hline
\end{tabular}

Porcentaje por filas. [Porcentaje por columnas]. (n). 


\section{Consumo de cannabis y tabaco}

En la tabla 5 se presenta la distribución de los participantes según el policonsumo (tabaco + cannabis). Para el análisis de la relación entre estas variables se agruparon los consumidores de cada una de las dos sustancias en una sola categoría, independientemente de la frecuencia de consumo. Se aprecia que a medida que aumenta la experiencia como fumador, disminuye el porcentaje de participantes sin experiencia con el cannabis y aumenta el porcentaje de aquellos que sí la han tenido. Las diferencias observadas son estadísticamente significativas $\left(X^{2}(9)=316.2 ; P<0.0005\right)$.

\section{Tabla 5: Distribución de los participantes en función del consumo de tabaco y cannabis.}

\begin{tabular}{|c|c|c|c|c|c|}
\hline Consumo de tabaco & $\begin{array}{c}\text { No he fumado } \\
\text { nunca un porro } \\
\text { entero }\end{array}$ & $\begin{array}{l}\text { He fumado un } \\
\text { porro o más } \\
\text { sólo por probar }\end{array}$ & $\begin{array}{c}\text { Antes fumaba } \\
\text { porros pero ya } \\
\text { no fumo }\end{array}$ & $\begin{array}{l}\text { Fumador ocasio- } \\
\text { nal o habitual de } \\
\text { porros }\end{array}$ & Total \\
\hline $\begin{array}{l}\text { No he fumado } \\
\text { nunca un cigarrillo } \\
\text { entero. }\end{array}$ & $\begin{array}{c}90.7 \% \\
{[48.5 \%]} \\
(127)\end{array}$ & $\begin{array}{c}7.1 \% \\
{[7.4 \%]} \\
(10)\end{array}$ & $\begin{array}{c}0.7 \% \\
{[1.8 \%]} \\
(1)\end{array}$ & $\begin{array}{l}1.4 \% \\
{[1.6 \%]} \\
(2)\end{array}$ & $\begin{array}{c}100 \% \\
{[24.2 \%]} \\
(140)\end{array}$ \\
\hline $\begin{array}{l}\text { He fumado un } \\
\text { cigarrillo o más sólo } \\
\text { por probar. }\end{array}$ & $\begin{array}{c}56.2 \% \\
{[34.7 \%]} \\
(91)\end{array}$ & $\begin{array}{c}35.2 \% \\
{[42,2 \%]} \\
\quad(57)\end{array}$ & $\begin{array}{c}1.2 \% \\
{[3.6 \%]} \\
(2)\end{array}$ & $\begin{array}{c}7.4 \% \\
{[9.5 \%]} \\
(12)\end{array}$ & $\begin{array}{c}100 \% \\
{[28 \%]} \\
(162)\end{array}$ \\
\hline $\begin{array}{l}\text { Antes fumaba } \\
\text { cigarrillos pero ya } \\
\text { no fumo. }\end{array}$ & $\begin{array}{c}19.7 \% \\
{[5.3 \%]} \\
(14)\end{array}$ & $\begin{array}{c}22,5 \% \\
{[11.9 \%]} \\
(16)\end{array}$ & $\begin{array}{c}33.8 \% \\
{[43.6 \%]} \\
(24)\end{array}$ & $\begin{array}{c}23.9 \% \\
{[13.5 \%]} \\
(17)\end{array}$ & $\begin{array}{c}100 \% \\
{[12,3 \%]} \\
(71)\end{array}$ \\
\hline $\begin{array}{l}\text { Fumador habitual } \\
\text { u ocasional de ciga- } \\
\text { rrillos. }\end{array}$ & $\begin{array}{c}14.6 \% \\
{[11.5 \%]} \\
(30)\end{array}$ & $\begin{array}{c}25.4 \% \\
{[38.5 \%]} \\
(52)\end{array}$ & $\begin{array}{c}13.7 \% \\
{[50.9 \%]} \\
(28)\end{array}$ & $\begin{array}{c}46.3 \% \\
{[75.4 \%]} \\
(95)\end{array}$ & $\begin{array}{c}100 \% \\
{[35.5 \%]} \\
(205)\end{array}$ \\
\hline Total & $\begin{array}{c}45.3 \% \\
{[100 \%]} \\
(262)\end{array}$ & $\begin{array}{c}23.4 \% \\
{[100 \%]} \\
(135)\end{array}$ & $\begin{array}{c}9.5 \% \\
{[100 \%]} \\
(55)\end{array}$ & $\begin{array}{c}21.8 \% \\
{[100 \%]} \\
(126)\end{array}$ & $\begin{array}{c}100 \% \\
{[100 \%]} \\
(578)\end{array}$ \\
\hline
\end{tabular}

Porcentaje por filas. [Porcentaje por columnas]. (n).

\section{DISCUSIÓN}

Más de las tres cuartas partes de los universitarios de la muestra y más de la mitad de las universitarias han fumado alguna vez un porro. Respecto al consumo habitual de esta droga, casi la mitad de los hombres y algo menos de la tercera parte de las mujeres son o han sido consumidores habituales de esta sustancia. Estos resultados son superiores a los hallados por otros investigadores en población universitaria (Bobes, et al., 2000) y se hallan en la línea de los informes del Ministerio de Interior (2003) respecto al sensible incremento del consumo de cannabis entre los jóvenes.

Si bien se observa que existen más consumidores de cannabis entre los varones que entre las mujeres, la magnitud de consumo de los que se reconocen consumidores es similar tal y como se ha detectado en estudios previos (Bobes et al., 2000).

Sorprende que son las mujeres las que se inician antes en el consumo, resultados éstos que difieren de los informados por el Ministerio del Interior (2002, 2003) y que nos hacen pensar en una posible inversión de la tendencia. En este sentido parece que aunque muchas más mujeres que hombres prefieren no pro- bar el cannabis, aquellas que deciden hacerlo lo hacen más precozmente que ellos y si continúan consumiendo lo hacen con la misma intensidad. No obstante, el reducido número de participantes masculinos de nuestro estudio nos hace ser prudentes en la interpretación de estos datos.

Otra cuestión a destacar es que la edad media de primera experiencia con el cannabis es sensiblemente superior entre aquellos que se han limitado a probarlo respecto a los que posteriormente se han convertido en fumadores habituales de la sustancia. Estos resultados hacen pensar que el riesgo de habituación se incrementará a medida que disminuya la edad de la primera experiencia con esta droga. Una posible explicación a este fenómeno podría ser que a edades más tempranas los adolescentes son más vulnerables a la presión del entorno, y principalmente de los iguales, y probablemente sea más fácil que una vez superada la curiosidad de la primera experiencia, no sepan negarse a continuar consumiendo cuando son invitados a ello. Esta hipótesis deberá ser contrastada en posteriores estudios. En cualquier caso, un factor protector que debe convertirse en objetivo prioritario de padres y educadores, es retrasar al máximo la edad de primer contacto experimental con la droga entre los adolescentes tal y como sugieren Becoña et al. (2004). 
En este sentido, una relación positiva y un estrecho vínculo afectivo con los padres podrían actuar como factores protectores para el consumo de drogas (Martínez y Robles, 2001; Martínez, Fuertes, Ramos y Hernández, 2003; Muñoz y Graña, 2001).

La creencia respecto a que el cannabis ayuda a relajarse y a desinhibirse está muy extendida entre los jóvenes, principalmente entre aquellos que han sido o son consumidores habituales de esta sustancia. Al tratarse de un estudio transversal no es posible saber si tener estas creencias potencia el consumo, o bien los consumidores de algún modo justifican el consumo por sus efectos beneficiosos. En cualquier caso, una buena parte de los no consumidores, tienen también estas creencias. De forma similar, Llorens, Perelló y Palmer (2004) en una investigación sobre estrategias de afrontamiento en estudiantes universitarios detectaron que los participantes que señalaban saber relajarse sin drogas, consumían un $78 \%$ menos de cannabis. En consecuencia, sería de interés que los programas de tratamiento y prevención incorporaran el aprendizaje de habilidades propias como puede ser la relajación. Asimismo, tal y como proponen Becoña et al. (2004), las propuestas de actuación a nivel de estrategias informativas futuras, deben facilitar la difusión de los hallazgos científicos sobre los cannabinoides a fin de evitar informaciones sesgadas y posteriormente analizar de qué forma esta información llega a los jóvenes de manera significativa y creíble.

Tal y como era de esperar, se observa una intensa relación entre la magnitud de consumo y la dependencia percibida. Son los mayores consumidores los que perciben una mayor dependencia. En la misma línea, en una investigación de Amigó y Seshadri (1999) con estudiantes de instituto y de universidad consumidores de cannabis se observó que al $28 \%$ le correspondería el diagnóstico de dependencia según el DSM -IV y asimismo el $17 \%$ reconoció algo de dependencia al cannabis.

Respecto al policonsumo tabaco-cannabis, se confirma la asociación descrita en otros estudios (Ministerio de Interior, 2002): nueve de cada diez participantes que nunca han fumado tampoco han consumido nunca cannabis, en cambio entre los fumadores de cigarrillos sólo 1.5 de cada diez se halla en esa situación. Estos elevados índices de policonsumo, tal y como proponen Bobes y Calafat (2000), sugieren la relevancia de que los abordajes terapéuticos tengan presente los diferentes consumos concomitantes, al tiempo que refuerzan una vez más la importancia de realizar campañas conjuntas contra el consumo de drogas, legales o ilegales, y están de acuerdo con la idea de que el consumo de tabaco, al margen de los perjuicios para la salud que supone, puede ser la puerta de entrada al consumo de otras drogas igual o mayor peligrosidad (Kandel, 1992).
En futuros estudios, teniendo en cuenta que la muestra estaba formada por estudiantes universitarios y que el consumo de cannabis en el período académico se asocia a notas peores, pobre rendimiento académico y una mayor prevalencia del abandono de los estudios, sería de interés analizar cómo son percibidos estos posibles efectos por parte de los adolescentes y jóvenes españoles (American Psychiatric Association, 2002; Fergusson, Horwood y Beautrais, 2003; Linskey, Coffey, Degenhardt, Carlin y Patton, 2003).

Además del reducido número de hombres de la muestra y las restricciones propias de un estudio transversal ya comentadas, otra limitación de este estudio es el sesgo de deseabilidad social inherente al uso de autoinformes. No obstante al haber garantizado el anonimato de los participantes, es de esperar que ese efecto no sea muy acusado.

\section{AGRADECIMIENTOS}

Este trabajo ha podido realizarse gracias a la ayuda para la dinamización y mantenimiento de la actividad investigadora de la UdG otorgado al grupo de investigación GRHCS52, año 2006.

\section{REFERENCIAS}

American Psychiatric Association (2002). Manual diagnóstico y estadístico de los trastornos mentales. Texto revisado. Barcelona Masson (edición original en inglés 2000)

Amigó, S. y Seshadri, V. (1999). Consumo de cannabis: determinantes y consecuencias en una muestra de estudiantes valencianos. Informació Psicològica, 71, 66-71.

Becoña, E., Calafat, A., del Cerro, J.A., et al. (2004). Informe sobre el cannabis 2004: análisis de situación y propuestas de actuación. [libro electrónico]. Recuperado el 15 de Junio de 2005, de http://www.msc.es/pnd/publica/ pdf/informe_cannabis2004.pdf

Becoña, E.; Palomares, A. y García, M.P. (1994). Tabaco y salud. Madrid: Pirámide.

Bobes, J., Bascarán, M.T., González, M.P. y Sáiz, P.A. (2000). Epidemiología del uso/abuso de cannabis. Adicciones, 12 (2), 31-40.

Calafat, A., Fernández, C., Becoña, E., Gil, E., Juan, M. y Torres, M.A. (2000). Consumo y consumidores de cannabis en la vida recreativa. Adicciones, 12 (2), 197-230.

Chabrol, H., Ducongé, E., Casas, C., Roura, C. y Carey, K.B. (2005). Relations between cannabis use and dependence, motives for cannabis use and anxious, 
depressive and borderline symptomatology. Addictive Behaviors, 30, 829-840.

Cunill, M., Planes, M. y Gras, M.E. (1998). Creencias sesgadas respecto al grado de "dureza" de algunas drogas en estudiantes universitarios. Adicciones, 10 (3), 233238.

Fergusson, D.M., Horwood, L.J. y Beautrais, A.L. (2003). Cannabis and educational achievement. Addiction, 98, 1681-1692.

Fernández, J., Álvarez, E., Secades, R., Jiménez, J.M., Cañada, A., Donate, I., Fernández, J.R. y Vallejo, G. (2003). Consumo de drogas de síntesis en estudiantes de secundaria del Principado de Asturias (España). Adicciones, 15 (1), 31-37.

Fuentes, J.A., Ruiz-Gayo, M., Manzanares, J., Vela, G., Reche, I. y Cordero, J. (1998). Potencialidad terapéutica de los cannabinoides y mecanismo de acción nocioceptiva. En J.L. Meana y L. Pantoja (Eds.). Derivados del cannabis: ¿drogas o medicamentos? (pp. 41-79). Bilbao: Universidad de Deusto.

Kandel, D.B. y Davies, M. (1992). Progresión to regular marijuana involvement: phenomenology and risk factors for near-daily use. En: M. Glantz y R. Pickens (Eds.). Vulnerability to drug abuse (pp. 211-253). Washington, DC: American Psychological Association.

Kandel, D.B. y Yamaguchi, K. (1985). Developmental patterns of the use of legal, illegal, and medically prescribed psychotropic drugs from adolescente to young adulthood. En: C.L. Jones y R.J. Battjes (Eds.). Etiology of drug abuse (pp.193-235). Rockville, MD: Nacional Institute on Drug Abuse.
Lynskey, M.T., Coffey, C., Degenhardt, L., Carlin, J.B. y Patton, G. (2003). A longitudinal study of the effects of adolescent cannabis use on high school completion. Addiction, 98, 685-692.

Llorens, N., Perelló, M. y Palmer, A. (2004). Estrategias de afrontamiento: factores de protección en el consumo de alcohol, tabaco y cannabis. Adicciones, 16 (4), 259264.

Martínez, J.M. y Robles, L. (2001). Variables de protección ante el consumo de alcohol y tabaco en adolescentes. Psicothema, 13 (2), 222-228.

Martínez, J.L., Fuertes, A., Ramos, M y Hernández, A. (2003). Consumo de drogas en la adolescencia: importancia del afecto y la supervisión parental. Psicothema, 15 (2), 161-166.

Megías, E. (2004) (Dir.). La percepción social de los problemas de drogas en España, 2004. Madrid: FAD.

Ministerio del Interior (2002). Observatorio Español sobre Drogas: Informe 5. [libro electrónico]. Recuperado el 13 de junio de http://www.mir.es/pnd/doc/observat/ estudios.htm .

Ministerio del Interior (2003). Observatorio Español sobre Drogas: Informe n6. [libro electrónico]. Recuperado el 13 de junio de http://www.mir.es/pnd/doc/observat/ estudios.htm .

Muñoz, M.J. y Graña, J.L. (2001). Factores familiares de riesgo y de protección para el consumo de drogas en adolescentes. Psicothema, 13 (1), 87-94.

Pinazo, S., Pons, J. y Carreras, A. (2002). El consumo de inhalables y cannabis en la preadolescencia: análisis multivariado de factores predisponentes. Anales de Psicología, 18 (1), 77-93. 